\title{
Prendre Dionysos par un détour. En hommage à Marcel
}

\section{Detienne}

Francesco Massa

\section{Citer ce document / Cite this document :}

Massa Francesco. Prendre Dionysos par un détour. En hommage à Marcel Detienne. In: ASDIWAL. Revue genevoise d'anthropologie et d'histoire des religions, $n^{\circ} 14,2019$. pp. 31-37;

doi : https://doi.org/10.3406/asdi.2019.1149

https://www.persee.fr/doc/asdi_1662-4653_2019_num_14_1_1149

Fichier pdf généré le 08/06/2020 


\section{Prendre Dionysos par un détour. En hommage à Marcel Detienne}

\section{Francesco Massa}

"Aucune lecture de la Grèce n'est innocente", ne cesse de rappeler Marcel Detienne'. Les Grecs - nous pourrions dire les mondes grec et romain plus généralement - sont depuis toujours l'objet de récupérations variées: I'exercice du retour à l'Antique s'opère dans des contextes politiques et culturels divers, mais il reste chargé d'opérations idéologiques alternant ou combinant récupérations identitaires, instances polémiques et tentatives de justification politique. On puise dans le réservoir de l'Antiquité - dans ses exemples définis comme paradigmatiques - afin de récupérer de nobles modèles qui puissent soutenir et expliquer les actions du présent. La déconstruction et la dénonciation de ces mécanismes demeurent sans doute l'un des aspects les plus intéressants du métier de l'antiquisant-e et le parcours de Marcel Detienne nous en a montré les résultats sur des terrains différents: la mythologie, le comparatisme, l'identité nationale, etc. ${ }^{2}$ Un tel enseignement est encore d'actualité pour une génération comme la mienne qui, arrivant à l'Université au début des années 2000, n'a pas vécu en direct les débats savants des années 1960-1990; une génération qui malgré les différences d'origines et de parcours a parfois choisi de travailler sur les mondes antiques après avoir lu les travaux, entre autres, de Jean-Pierre Vernant, Pierre Vidal-Naquet, Marcel Detienne et Nicole Loraux. À cela s'ajoute un constat formel: relire les textes de Marcel Detienne, c'est toujours une découverte et une célébration de la pensée. La richesse de sa langue ne cesse de surprendre et de stimuler la réflexion: les expressions qu'il choisit, les adjectifs qu'il attribue aux mots, les tournures des phrases rendent ses travaux quasiment uniques dans le panorama des études anciennes.

Parmi les centres d'intérêt qui ont animé les recherches de Marcel Detienne, Dionysos occupe assurément une place de choix. Personnage "mobile» et "mouvant», le dieu offre la clé privilégiée d'un dépaysement que Detienne cherchait (et qu'il invitait à chercher) chez les

Je tiens à remercier les collègues et ami-e-s qui ont accepté de relire ce texte et de partager leur avis avec moi: Philippe Borgeaud, Dominique Jaillard, Renée Koch Piettre et Vinciane Pirenne-Delforge.

1 Marcel Detienne, Dionysos mis à mort, Paris, Gallimard, 1996², p. 17 (orig. 1977).

2 Sur ces différents domaines, voir les textes de Philippe Borgeaud, "Hommage à Marcel Detienne ", Kernos 32 (2019), pp. 9-12 et ici même, ainsi que Renée Koch Piettre, Arnaud Sérandour, "Hommage à Marcel Detienne", Semitica et Classica 12 (2019), pp. 307-310. 
Grecs. Les deux ouvrages centrés sur cette figure emblématique du monde divin ancien, le Dionysos mis à mort publié en 1977 et le Dionysos à ciel ouvert publié en 1986, constituent un diptyque publié en dix ans. Pour celui qui avait d'abord lu ces textes en traduction italienne, la découverte des originaux français fut celle d'une explosion sémantique, une célébration de la pensée vive. Ces quelques pages n'ont pas pour but d'évaluer la "véridicité " des travaux que Marcel Detienne a consacrés au dieu Dionysos; que I'on soit d'accord ou non avec ses interprétations, ce qui importe ici est de souligner que ses ouvrages ont marqué un tournant dans I'historiographie des années 1970 et 1980, en contribuant à renouveler les études sur les polythéismes antiques.

Lorsque Detienne commence à s'intéresser à la figure de Dionysos, la bibliographie sur ce dieu a déjà connu des évolutions importantes. Une année en particulier représente une date charnière dans I'historiographie: en 1951, deux ouvrages séminaux voient le jour, The Greeks and the Irrational d'Eric R. Dodds et Dionysos. Histoire du culte de Bacchus d'Henri Jeanmaire ${ }^{3}$. Le volume de Dodds contenait en appendice un essai sur le ménadisme qui, pour la première fois, proposait une interprétation de la mania dionysiaque à travers la comparaison avec des phénomènes religieux modernes, en insistant notamment sur la dimension "psychologique " du culte dionysiaque; et l'ouvrage de Jeanmaire s'ouvrait de manière novatrice aux analyses des ethnographes, des anthropologues et des psychiatres qui étaient utilisées pour expliquer les phénomènes bachiques ${ }^{4}$. Leurs réflexions essayaient de sortir du "problème des origines " qui animait le débat savant depuis le XIX ${ }^{e}$ siècle. Les spécialistes de ce temps étaient soucieux de comprendre l'origine du dieu et d'expliquer comment il était arrivé en Grèce. Dans ce cadre, ils considéraient Dionysos comme une divinité non hellénique, au moins depuis l'ouvrage de Friedrich Creuzer, Dionysus, paru en 1809. Dans ce texte qui est souvent signalé comme étant la première monographie dionysiaque, le dieu était présenté comme une divinité non grecque, d'origine égyptienne ${ }^{5}$. Plus tard, l'important travail d'Erwin Rohde, Psyche, de 1884, confirma la nature étrangère en proposant en revanche une origine thrace ${ }^{6}$. Une telle hypothèse fut encore proposée au début $\mathrm{du} x \mathrm{x}^{\mathrm{e}}$ siècle par Franz Cumont qui définit Dionysos comme un dieu "à demi oriental» dans son appendice à son livre sur Les religions orientales dans le paganisme romain. D'après Cumont, néanmoins, l'identité dionysiaque était plus complexe: Dionysos était bien d'origine thrace, mais sa nature orientale n'était pas une conséquence de son origine: il l'avait davantage développée à la suite de la diffusion de son culte dans le monde hellénistique, dans le sillage des conquêtes d'Alexandre, et de son identification «avec divers dieux indigènes"; ainsi, lorsqu'elles furent introduites en Italie, les pratiques bachiques

3 Eric R. Dodds, The Greeks and the Irrational, Berkeley - Los Angeles, University of California Press, 1951, et HenRI JeanmalRe, Dionysos. Histoire du culte de Bacchus, Paris, Payot, 1951.

4 ERIC R. Dodds, "Maenadism in the Bacchae», Harvard Theological Review 33 (1940), pp. 155-176. Sur l'importance de Dodds dans I'histoire des études sur Dionysos, voir notamment Albert Henrichs, "Loss of Self, Suffering, Violence: The Modern View of Dionysos from Nietzsche to Girard", Harvard Studies in Classical Philology 88 (1984), pp. 205240. Plus généralement sur le ménadisme, voir Albert HenRichs, "Greek Maenadism from Olympias to Messalina ", Harvard Studies in Classical Philology 82 (1978), pp. 121-160 et JAN N. BrEMmER, "Greek Maenadism Reconsidered", Zeitschrift für Papyrologie und Epigraphik 55 (1984), pp. 267-286.

5 Georg Friedrick Creuzer, Dionysus, sive Commentationes academicae de rerum Bacchicarum orphicarumque originibus et causis, Heidelberg, Mohr und Zimmer, 1809, IV, chap. I, § 4. L'hypothèse fut ensuite reprise dans Symbolik und Mythologie der alten Völker, Leipzig - Darmstadt, K. W. Leske, 1810-1812.

6 Erwin Rohde, Psyché. Le culte de l'âme chez les Grecs et leur croyance à l'immortalité, Paris, Payot, 1952, p. 267s. (orig. Breisgau - Leipzig, Mohr, 1890-1894). 
étaient un "culte composite, plus ou moins asiatique ou égyptien " ${ }^{7}$. Derrière cette quête se cachait l'idée que l'identification géographique des origines aurait permis à elle seule de définir l'"essence» du dieu. Cette approche n'était pas limitée aux études sur Dionysos; au contraire, les travaux des antiquisants se situaient dans ce que Marc Bloch appelait "l'idole des origines ", un fantasme qui touchait une grande partie de l'historiographie de cette époque ${ }^{9}$.

Une telle approche était destinée à être définitivement remise en cause peu de temps après la publication des livres de Dodds et Jeanmaire: entre 1952 et 1953, en effet, la découverte de l'origine grecque du linéaire B, proposée par Michael Ventris et John Chadwick, permit aux chercheurs de repenser la question des origines de la culture grecque. Un peu plus tard le nom de Dionysos fut identifié sur des tablettes découvertes à Pylos, ce qui attesta la présence du dieu en Grèce depuis l'époque mycénienne ${ }^{10}$.

Sur ces bases, dans les années 1970 deux opérations intellectuelles nouvelles se sont inscrites dans l'histoire des études: d'une part, celle de Károly Kerényi qui s'enracine dans la pensée jungienne pour faire du dieu grec un archétype de l'existence humaine ${ }^{11}$; d'autre part, celle de Marcel Detienne. Les deux lectures prennent des voies opposées, comme l'a bien montré Albert Henrichs dans un article de référence sur I'historiographie dionysiaque ${ }^{12}$ : si Kerényi exaltait la puissance naturelle de Dionysos, sa manière d'irriguer la vie des êtres humains, Detienne insistait sur la "marginalité» du dieu. En 1977, Dionysos mis à mort représente la tentative d'expliquer l'altérité du dieu qui ne faisait pas partie de l'Olympe d'Homère. L'élément central du volume était la nature "insaisissable» de Dionysos qui se définissait notamment par des couples d'oppositions (centre et périphérie, humain et bestial, folie et sagesse, ivresse et sobriété, accueil et rejet) ${ }^{13}$.

Dans le Dionysos mis à mort, c'est le sacrifice qui est au centre de la réflexion et la manière dont la ritualité dionysiaque exprime une forme de contestation du modèle sacrificiel civique ${ }^{14}$. Plus particulièrement, c'est la forme sanglante qui intéresse l'auteur et notamment son rapport avec des pratiques qui se rapprochent du meurtre. Detienne situe Dionysos dans une configuration comportant quatre systèmes aux marges de la religion de la polis: pythagorisme

7 Franz Cumont, Les religions orientales dans le paganisme romain, Corinne Bonnet et Françoise Van Haeperen éds., Turin, Nino Aragno, 2006, p. 316 (orig. Paris, Annales du Musée Guimet, 1906, 4éd. 1929). Sur cet aspect, voir FrANCESCO MASSA, "Pourquoi Bacchus? L'appendice sur les mystères bachiques à Rome dans la construction herméneutique des Religions orientales de Franz Cumont", in Annelies Lannoy, Danny Praet éds., The Christian Mystery. Early Christianity and the Pagan Mystery Cults in the Work of Franz Cumont (1868-1947) and in the History of Scholarship, Stuttgart, Franz Steiner, à paraître.

8 Il suffit de penser, toujours dans le contexte dionysiaque, au débat sur les origines de la tragédie athénienne. Sur la question des origines dans les études sur l'Antiquité, voir DiEgo LANZA, "La tragedia e il tragico ", in Salvatore Settis éd., I Greci, vol. I, Turin, Einaudi. pp. 469-505.

9 MARC Bloch, Apologie pour I'histoire ou métier de I'historien, Paris, Armand Colin, 1952², pp. 5-9 (orig. 1949).

10 Sur le déchiffrement du linéaire B, voir JOHN CHADWICK, The Decipherment of Linear B, Cambridge, Cambridge University Press, 1958; sur les tablettes mycéniennes portant le nom de Dionysos, voir Alberto Bernabé, "Dionysos in the Mycenaean World", in Alberto Bernabé et al. éds., Redefining Dionysos, Berlin - Boston, De Gruyter, 2013, pp. 23-37.

11 Voir Károly Kerényı, Dionysos. Urbild des unzerstörbaren Lebens, München - Wien, Langen Müller, 1976. II s'agit d'une publication posthume, mais plusieurs articles avaient anticipé les points forts de cet ouvrage déjà dans les années 1950-1960. Sur le Dionysos de Kerényi, voir PAOLA PISI, "Dioniso da Nietzsche a Kerényi », Studi e Materiali di Storia delle Religioni 69.1 (2003), pp. 129-218, en particulier pp. 196-204.

12 Albert Henrichs, "Loss of Self, Suffering, Violence», p. 210.

13 Je n'aborde pas ici la méthode structuraliste de Detienne qui a fait l'objet de critiques féroces à la suite de la publication de son ouvrage et qui a été analysée à plusieurs reprises; voir par exemple RENÉE Koch PIETTRE, "Les Grecs et nous. L'anthropologie comparative de Marcel Detienne ", Mètis n.s. 6 (2008), pp. 345-376.

14 Marcel Detienne, Dionysos mis à mort, p. 8. 
et orphisme; dionysisme et cynisme ${ }^{15}$. Ces quatre "chemins de déviance" ont pour but de protester contre le système de la cité, par le haut ou par le bas, vers les dieux ou vers les bêtes; mais tous visent au même objectif, dépasser les modèles religieux civiques ${ }^{16}$. Dans la dernière section de l'ouvrage, Dionysos orphique et le bouilli rôti, Detienne se concentre plus particulièrement sur la stratégie adoptée par le dionysisme. Pour expliquer le choix effectué par les adeptes de Dionysos, il se concentre sur le récit de la mise à mort du dieu par les Titans, dont la reconstruction et l'interprétation sont particulièrement complexes, comme le montrent les nombreuses études récentes consacrées à ce sujet ${ }^{17}$. En se fondant sur une affirmation tirée des Problèmes du Pseudo-Aristote, Detienne reconnaît dans ce mythe une inversion de la procédure sacrificielle courante, qui prescrivait de rôtir les chairs d'abord et de les bouillir ensuite. Voici le texte en question:

Pourquoi n'est-il pas permis de rôtir le bouilli, alors que ce l'est de bouillir le rôti? Est-ce à cause de ce qui est dit dans la Teletè, ou bien est-ce parce que les hommes apprirent plus tard à préparer les mets bouillis, car autrefois ils rôtissaient tout? Assurément, rôtir le bouilli, ce serait revenir en arrière..$^{18}$

Dans la lecture proposée par Detienne, c'est du Dionysos orphique qu'il est question dans le Problème aristotélicien: la Teletè ne serait donc que l'un des écrits d'Orphée rapportant I'histoire du Dionysos "orphique». En considérant l'inversion entre bouilli et rôti comme une caractéristique du mythe du sacrifice de Dionysos, Detienne précise son interprétation du récit bachique:

En adoptant le schème «bouilli suivi de rôti », les Orphiques entendent bien nier le procès qui fait du sacrifice, au niveau de la cuisine, un acte positif, une pratique à connotation "progressive ». Aller du bouilli au rôti, ou rôtir le bouilli, c'est, tout en respectant l'apparence formelle du sacrifice, l'inverser du dedans, le détruire de l'intérieur après l'avoir condamné du dehors. Le sacrifice est un mal; rien ne peut infléchir son orientation funeste. ${ }^{19}$

15 Ibid., p. 145.

16 Je ne discute pas ici de l'importance du livre de Dario SabBatuccl, Saggio sul misticismo greco, Roma, Edizioni dell'Ateneo, 1965, dans la construction de Detienne; je me limite à renvoyer à ALESSANDRO TESTA, Miti antichi e moderne mitologie. Saggi di storia delle religioni e storia degli studi sul mondo antico, Roma, Aracne, 2010, pp. $334-355$ et ID., "Dioniso nelle mitologie francesi e italiane», Limes. Revista de estudios clásicos 25 (2012), pp. 89-115.

17 Voir, en ne citant que des essais récents, RadCLIFFE Edmonds, "Tearing apart the Zagreus Myth: a Few Disparaging Remarks on Orphism and Original Sin», Classical Antiquity 18.1 (1999), pp. 35-73; Alberto Bernabé, "La toile de Pénélope: a-t-il existé un mythe orphique sur Dionysos et les Titans? ", Revue de l'histoire de religions 219.4 (2002), pp. 401-433; ID., "Autour du mythe de Dionysos et les Titans. Quelques notes critiques», in DomENICO AcCORINTI, Pierre Chuvin éds., Des Géants à Dionysos, Mélanges de mythologie et de poésie grecque offerts à Francis Vian, Alessandria, Dell'Orso, 2003, pp. 25-39; Noel Robertson, "Orphic Mysteries and Dionysiac Ritual», in Michael B. Cosmopoulos éd., The Greek Mysteries: Archaeology and Ritual of Ancient Greek Secret Cults, London - New York, Routledge, 2003, pp. 218-240; Miguel Herrero De JÁuregui, "Dionysos mi-cuit: I'étymologie de Mésatis et le festin inachevé des Titans", Revue de l'histoire des religions 223.4 (2006), pp. 389-416; Renaud Gagné, Ancestral Fault in Ancient Greece, Cambridge, Cambridge University Press, 2013, pp. 455-460.

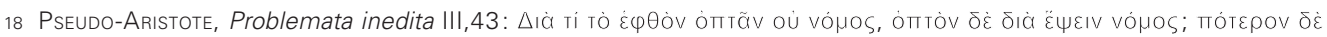

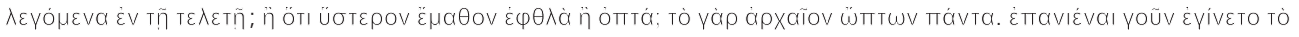
$\dot{\varepsilon} \varphi \theta$ òv kà̀ ómTã V.

19 Marcel Detienne, Dionysos mis à mort, p. 188 
L'interprétation proposée par Detienne ne semble guère tenir la route une fois que l'on procède à une analyse détaillée des sources ${ }^{20}$. Néanmoins, il est plus important de situer cette lecture du mythe de la mise à mort de Dionysos sur l'horizon plus large envisagé par Detienne. Par-delà l'analyse philologique, l'enjeu le plus important du livre était de réfléchir à la valeur de la pratique sacrificielle et aux détails des gestes rituels. Et Dionysos est utilisé à cette fin.

C'est pourquoi le dieu est défini comme étant la "synthèse d'une histoire qui commençait avec la sauvagerie des Peuples de la Nature et s'achevait dans la maturité spirituelle de la religion chrétienne, centrée sur un dieu personnel, immolé parce qu'il se sacrifie $»^{21}$. Nous décelons dans ces pages l'influence de la Religionsgeschichtliche Schule allemande dans sa définition des rapports entre christianisme et religions traditionnelles. Dans cette perspective, Dionysos apparaît «à la confluence des religions anciennes du Proche-Orient et de la nouvelle doctrine de salut, affirmée par le christianisme $»^{22}$.

Si le Dionysos mis à mort de 1977 avait analysé le sacrifice de Dionysos et les pratiques sanglantes qui l'entouraient, dix ans plus tard Detienne ouvre son nouveau livre dionysiaque en définissant Dionysos comme un "dieu vivant». Dans le Dionysos à ciel ouvert de 1986 titre qui fait allusion à un sanctuaire gaulois dont le toit était monté et démonté tous les ans -, Detienne insiste sur le fait que Dionysos est le dieu le plus cosmopolite et le moins sédentaire; il réfléchit sur sa présence dans plusieurs cités de la Méditerranée, sous des formes et des noms différents. Sans la désigner explicitement, la première partie du livre réfléchit à la question de l'interpretatio du dieu, par le biais de la notion de "dieu épidémique». L'expression est liée à la dimension de la théophanie, et plus précisément à l'arrivée d'un dieu de l'extérieur et aux sacrifices qui sont offerts aux divinités lorsqu'elles "arrivent dans le pays, quand elles se rendent dans un sanctuaire $»^{23}$. Les dieux "migrateurs» sont honorés par ces épidémies; et il convient de reconnaître, en effet, que dans les récits mythiques antiques Dionysos est le « dieu migrant" par excellence, celui qui traverse les frontières, en tant qu'exilé ou conquérant. La "pulsion épidémique » du dieu s'exprime dans les récits qui mettent en scène son arrivée, que ce soit à Thèbes, à Ikaria, à Lesbos ou en Inde. Dionysos est celui qui voyage dans l'espace méditerranéen en apportant la culture de la vigne et en apprenant aux hommes à produire le vin. Detienne questionne l'image de Dionysos comme der kommende Gott, "le dieu qui vient », une expression née dans le contexte allemand de la fin $d u X \|_{I I}{ }^{e}$ et du début du XIX $x^{e}$ siècle. La quête d'une "nouvelle mythologie» guidait les réflexions de Johann Gottfried Herder et de Friedrich Wilhelm Schelling, et Dionysos incarnait la puissance qui permettait de dépasser la civilisation

20 Detienne ne souligne pas que cette inversion dans le mythe de Dionysos et les Titans n'est attestée que dans Clément d'Alexandrie, Protreptique aux Hellènes II,17,2-18,2 et qu'elle n'est pas présente ailleurs: voir par exemple Diodore III,62,6; Hygin, Fables 167,1; Plutaroue, Manger de la viande I,7,996C. Voir Pierre Ellinger, La légende nationale phocidienne. Artémis, les situations extrêmes et les récits de guerre d'anéantissement, Paris, De Boccard, 1993, pp. 147-195, qui revient sur les propositions de M. Detienne. J'ai moi-même analysé l'interprétation de Detienne dans Francesco MASSA, Tra la vigna e la croce. Dioniso nei discorsi letterari e figurativi cristiani (II-IV secolo), Stuttgart, Franz Steiner, 2014, pp. 99-105.

21 Marcel Detienne, Dionysos mis à mort, p. 9.

22 Ibid., pp. 164-165.

23 Marcel Detienne, Dionysos à ciel ouvert, Paris, Hachette, 1986, p. 12. Voir aussi ID., "Dionysos et ses parousies: un dieu épidémique ", in L'association dionysiaque dans les sociétés anciennes, Roma, École française de Rome, 1986, pp. 53-83. 
moderne, dépourvue d'une mythologie propre ${ }^{24}$. À partir d'un tel noyau savant, Detienne donne un tout autre sens au "dieu qui vient», en construisant l'une des idées les plus fécondes sur la valeur de l'expérience dionysiaque. Tout en interrogeant la question de la diffusion des cultes dionysiaques, dans le Dionysos à ciel ouvert il refuse d'adopter un paradigme diffusionniste pour expliquer le succès du dionysisme, pourtant à la mode à l'époque et attesté par exemple dans l'œuvre déjà citée de K. Kerényi. L'épidémie devient en revanche un outil pour interpréter les discours, les rites et les croyances du monde dionysiaque dans le cadre des sociétés antiques.

Cette représentation d'un Dionysos en mouvement contribue également à construire l'image du dieu comme "l'étrange Étranger $»^{25}$. Une fois de plus, Detienne visait à montrer la face déroutante des pratiques grecques, leur dimension menaçante, sinon violente. II ne faut pas oublier qu'entre la publication du Dionysos mis à mort et celle du Dionysos à ciel ouvert en 1986, un autre Dionysos s'était établi au centre de la réflexion parisienne. II s'agit du "Dionysos masqué " de Jean-Pierre Vernant qui se situait dans l'Athènes du ve siècle avant notre ère et plus particulièrement dans la dernière pièce d'Euripide, les Bacchantes ${ }^{26}$. Le Dionysos à ciel ouvert paraît à la fois une tentative de dialogue et une réponse au Dionysos de Vernant, comme Marcel Detienne lui-même le reconnaît dans l'entretien qu'il a accepté de donner à notre revue Asdiwal en 2008:

Mon Dionysos est un Dionysos "dialoguant» avec le Dionysos de Vernant; son Dionysos, à lui, était beaucoup trop sage; il était rentré à Athènes, il s'était installé à la mairie, il célébrait les mariages. Moi, je considérais que Dionysos, c'était autre chose, un dieu qui avant tout faisait «péter les plombs » pour ainsi dire. Gommer l'effervescence, c'était châtrer Dionysos. ${ }^{27}$

Même si l'attention de Vernant se concentrait notamment sur l'Athènes de l'époque classique, les deux interprétations sont moins éloignées l'une de l'autre que Detienne ne le laisse entendre dans le passage cité. Vernant reprenait lui aussi le thème de l'altérité comme composante essentielle du Dionysos euripidéen. Certes, cette altérité n'était plus examinée dans les marges ou dans les chemins de déviance, mais au cœur même de la cité et de ses pratiques rituelles ${ }^{28}$. Par-delà les histoires personnelles et les fractures humaines, il me semble que I'heure est venue de réfléchir davantage aux points de contact entre les interprétations de Detienne et de Vernant, et de souligner que la place centrale accordée aux rites caractérise et rapproche les analyses des deux savants.

24 Sur cette épiphanie dionysiaque dans la culture allemande, voir HANS G. KIPPENBERG, À la découverte de I'histoire des religions. Les sciences religieuses et la modernité, Paris, Salvator, 1999, pp. 189-199 (orig. Münich, C. H. Beck, 1997). Sur la "nouvelle mythologie» et le "dieu qui vient », voir MAnfred Frank, Der kommende Gott, Frankfurt, Suhrkamp, 1982 et Natale Spineto, "Tradizione e miti dionisiaci. Epifanie e ritorni del "Dio che viene" ", Storiografia 2 (1998), pp. 115-128, en particulier pp. 124-125.

25 Marcel Detienne, Dionysos à ciel ouvert, pp. 21-27. Voir déjà lD., Dionysos mis à mort, p. 163 où il était question de la "vocation pour l'Étrange» de Dionysos.

26 Jean-Pierre Vernant, "Le Dionysos masqué des Bacchantes d'Euripide", L'Homme 93 (1985), pp. 31-58; republié dans Jean-Pierre Vernant, Pierre Vidal-Naquet, Mythe et tragédie en Grèce ancienne, t. II, Paris, La Découverte, 1986, pp. 237-270.

27 Marcel Detienne, "Entretien", Asdiwal 3 (2008), pp. 13-22.

28 Vernant avait travaillé sur la figure de Dionysos dans le cadre de ses cours au Collège de France en 1983-1984: le résumé du cours fut publié dans Jean-Pierre Vernant, Figures, idoles, masques, Paris, Juillard, 1990, pp. 208-247. II reviendra encore sur Dionysos dans Mythe et religion en Grèce ancienne, Paris, Seuil, 1999, pp. 96-105 (le texte fut d'abord publié en anglais sous le titre "Greek Religion", in MiRCEA Eliade éd., The Encyclopedia of Religion, New York - London, Macmillan, 1987, vol. VI, pp. 99-118). 
Dans les années qui ont suivi la publication du Dionysos à ciel ouvert, Marcel Detienne a continué de travailler sur cette puissance divine, y revenant souvent dans ses recherches sur le polythéisme grec et son langage, son fonctionnement, ses rouages ${ }^{29}$. Non seulement dans le prolongement des recherches sur Orphée ${ }^{30}$, mais aussi et surtout dans son analyse du couple Apollon-Dionysos. Par-delà le paradigme nietzschéen, Detienne se propose de comprendre les liens entre les deux frères divins. Son regard se concentre d'abord sur le sanctuaire de Delphes où Apollon et Dionysos partageaient l'espace de l'oracle, mais il insiste sur l'importance de quitter le sanctuaire panhellénique pour comprendre la proximité entre les deux divinités ${ }^{31}$. II analyse donc d'autres lieux, Ikarion, Magnésie sur Méandre, Rhodes, en nous montrant une fois de plus que si l'on veut comprendre le fonctionnement de la religion grecque, il faut quitter les sentiers battus pour aller fouiller les interstices.

Dans la Postface du Dionysos mis à mort, rédigée à Johns Hopkins University en 1997, à l'occasion de la nouvelle édition du livre, l'auteur est plus explicite quant à la volonté polémique de son livre qui visait à réagir contre une représentation trop lisse de l'Antiquité et contre l'idée que l'étude des mondes grecs donnerait accès "directement et universellement à l'humain et à l'universel »32. L'opposition qu'il construit entre "le parti des adorateurs de la Grandeur de l'Antique» et "la foule grandissant de par le monde de ceux qui se rassemblent sous le signe du "Post" " (postmodernisme, postféminisme, postdéconstructionnisme) est encore d'actualité ${ }^{33}$. Dans des termes différents, les promoteurs des critiques les plus virulentes contre les réflexions nourries par les courants de pensée du "Post» cachent souvent des agendas essentialistes visant à faire de l'Antique un terrain de récupérations identitaires et théologiques.

\author{
Francesco Massa \\ Université de Fribourg \\ Institut du monde antique et byzantin \\ Rue Pierre-Aeby 16 \\ 1700 Fribourg \\ Suisse \\ francesco.massa@unifr.ch
}

\footnotetext{
29 Voir notamment Marcel Detienne, "Du polythéisme en général», Classical Philology 81 (1986), pp. 47-55 et ID., "Expérimenter dans le champ des polythéismes», Kernos 10 (1997), pp. 52-72.

30 Voir notamment Marcel Detienne, Les dieux d'Orphée, Paris, Gallimard, 1989.

31 Marcel Detienne, "Apollon und Dionysos in der griechischen Religion», in Richard Faber, Renate Schlesier éds., Die Restauration der Götter. Antike Religion und Neo-Paganismus, Würzburg, Königshausen - Neumann, 1986, pp. 124132 et ID., "Oublier Delphes entre Apollon et Dionysos ", Gradhiva 24 (1998), pp. 11-20.

32 Marcel Detienne, Dionysos mis à mort, p. 222.

33 Ibid., p. 223.
} 